\title{
Commentary on the superconducting and magnetic properties of the ruthenocuprates
}

Piotr W Klamut

Correspondence: P.Klamut@int.pan. wroc.pl

Institute of Low Temperature and Structure Research, Polish Academy of Sciences, P Nr 1410, 50-950

Wrocław 2, Poland

\begin{abstract}
Presented in this commentary is a short discussion of the superconducting and magnetic properties of selected compounds of ruthenocuprates, with an emphasis given to the $\mathrm{RuSr}_{2} \mathrm{GdCu}_{2} \mathrm{O}_{8}$ compound. The ruthenocuprates, which form derivative class in the cuprate family, continue raising interest for there evidenced presence of magnetic ordering in the sublattice of Ru ions, which in polycrystalline samples of $\mathrm{RuSr}_{2} \mathrm{GdCu}_{2} \mathrm{O}_{8}$ sets at $T_{m} \approx 132 \mathrm{~K}$, and at low temperatures may become simultaneous with the superconducting phase $\left(T_{c, \text { max }} \approx 50 \mathrm{~K}\right)$-all that in the crystal structure derived from well known $\mathrm{REBa}_{2} \mathrm{Cu}_{3} \mathrm{O}_{7}$ cuprate superconductor. While several here invoked recent results advocate for an intrinsically inhomogeneous nucleation of superconductivity within this structure, the formed superconducting phase carries potential of maintaining substantial anisotropy with then interesting consequence for probing the cuprate characteristic superconductivity in these compounds.

PACS Codes: 74.72.-h, 74.25. $\mathrm{Ha}$, 74.81.-g
\end{abstract}

Discovered in late eighties, ruthenocuprates continued raising interest for an apparent coexistence of their component magnetism in the Ru sublattice with the superconducting phase formed at lower temperatures. For polycrystalline samples of $\mathrm{RuSr}_{2} \mathrm{GdCu}_{2} \mathrm{O}_{8}$ the temperature of this ordering is $T_{m} \approx 132-136 \mathrm{~K}$, whereas the maximal temperature of superconducting transition was reported at approximately $45 \mathrm{~K}$ [1-3]. The magnetic ordering seems to acquire an effective ferromagnetic character at the magnetic field values well below the second critical field for the superconducting phase, which put together with fact that the ordering originates in $d$-type electron shells of the $\mathrm{Ru}$ ions, which also contribute electrons to the density of states close to Fermi level, have outlined broad interest in the properties of these compounds. Since the mechanism as well as prerequisite electronic structure necessary to achieve the superconducting condensation in cuprates, especially for charge underdoped part of the class phase diagram, are still at debate, the ruthenocuprates seemed offering a promising research platform not only to approach the issue of apparent coexistence of their transition metal magnetism with superconductivity but also to extend the investigation of cuprate characteristic superconductivity to a new domain of its possible coexistence with ferromagnetism. Several years of intensive research provided us with a lot of information, however, as we learn more, a handful of questions still remain unanswered concerning very nature of complex properties of these compounds. The challenge remains to provide a compelling description of the magnetic ground state, to elucidate a role of the

(c) 2010 Klamut This is an open access article distributed under the terms of the Creative Commons Attribution License (http:// creativecommons.org/licenses/by/2.0), which permits unrestricted use, distribution, and reproduction in any medium, provided the original work is properly cited. 
submicro-and nanometre range structural alternations for supporting/affecting the superconducting condensate, and to learn details of the internal charge transfer between the $\mathrm{Ru}-\mathrm{O}$ and $\mathrm{Cu}-\mathrm{O}$ electronic subsystems, that at least for its potentially decisive role in determination of electronic and magnetic properties of the compounds.

For description of the crystal structure of $\mathrm{RuSr}_{2} \mathrm{GdCu}_{2} \mathrm{O}_{8}$ is instructive to compare it with $\mathrm{CuBa}_{2} \mathrm{GdCu}_{2} \mathrm{O}_{7}$, which represents the well known 123-type cuprate formula rewritten for distinguishing two crystallographically distinct positions of the $\mathrm{Cu}$ ions. First $\mathrm{Cu}$ symbol corresponds there to the so called chain position, which is coordinated with four oxygens, two of which are in the $a-b$ plane and two other are collinear with that $\mathrm{Cu}$ ion along the $c$ direction. It is of the structural layers which contain such chains to provide for substantial decrease of the anisotropy of superconducting phase in the 123-type cuprate, which comes in expectance of the proximity effect induced superconducting condensate (the condensate anisotropy in cuprate structure is expected in a sense of the anisotropy of its characteristic coherence lengths $\zeta_{c}<\zeta_{\mathrm{ab}}$ with $\zeta_{c}$ comparable to structural thickness of the $\mathrm{Cu}-\mathrm{O}$ layers). For $\mathrm{RuSr}_{2} \mathrm{GdCu}_{2} \mathrm{O}_{8}$, the $\mathrm{Cu}$ chain position should replace with the $\mathrm{Ru}$ ion then coordinated with two additional planar oxygens in forming the layer of corner sharing $\mathrm{RuO}_{6}$ octahedra along the $a-b$ planes of the crystal. Remaining part of the structure is analogous to that of the 123-type compound. The Ru-O layers are then intertwined with structural blocks composed of two neighbouring $\mathrm{Cu}-\mathrm{O}(2)$ planes with single layer of $\mathrm{RE}^{3+}$ ions positioned in between them and two outer layers of $\mathrm{Sr}^{2+}$ ions (in the analogous position to that of $\mathrm{Ba}^{2+}$ in $\left.\mathrm{REBa}_{2} \mathrm{Cu}_{3} \mathrm{O}_{7}\right)$ and $\mathrm{O}(4)$ oxygens. The $\mathrm{O}(4)$ position is apical for both the $\mathrm{RuO}_{6}$ octahedron and the $\mathrm{CuO}_{5}$ pyramid (its base is in the $\mathrm{Cu}-\mathrm{O}(2)$ plane). Then, the shift of bridging $\mathrm{O}(4)$ ions or other modification of distances in the $\mathrm{Cu}-\mathrm{O}(4)-\mathrm{Ru}$ bonds should influence the intra-structural charge transfer between the $\mathrm{Ru}-\mathrm{O}$ and $\mathrm{Cu}-\mathrm{O}$ layers. The $\mathrm{Cu}-\mathrm{O}(4)$ and $\mathrm{Ru}-\mathrm{O}(4)$ bond lengths, as it was determined in the neutron powder diffraction experiment described in [4], have mutually inverse temperature dependence and become equal at the temperature approximately matching $T_{m}$ (the $c$ axis rotations of $\mathrm{RuO}_{6}$ octahedra and the $\mathrm{Ru}-\mathrm{O}(4)$ distance both increase on cooling). Below similar characteristic temperature were also mapped fixing of the buckling angle in the $\mathrm{Cu}-\mathrm{O}$ planes (defined as departure from $180^{\circ}$ of the $\mathrm{Cu}-\mathrm{O}(2)-\mathrm{Cu}$ planar bond) and of the distance between neighbouring $\mathrm{Cu}-\mathrm{O}$ planes [4]. Also at the temperature close to $T_{m}$ the $c / a(T)$ dependence modifies from decreasing to increasing on lowering the temperature, what may lead to the associated modification of charge doping in the $\mathrm{Cu}-\mathrm{O}$ planes and $\mathrm{Ru}$-O layers [5]. The synchrotron X-ray diffraction performed for $\mathrm{RuSr}_{2} \mathrm{Gd}$ $\mathrm{Cu}_{2} \mathrm{O}_{8}$ has determined presence of the structural distortions in form of approx. $14^{\circ}$ rotations of the $\mathrm{RuO}_{6}$ octahedra along with their slight tilting off the $c$ axis, which may reflect from accommodation of the planar $\mathrm{Ru}-\mathrm{O}$ bonds in layered structure. The distortions were found to promote the superstructure appearing with characteristic domain size in between 5 and $20 \mathrm{~nm}$ [6]. Symmetry of the $\mathrm{RuSr}_{2} \mathrm{GdCu}_{2} \mathrm{O}_{8}$ structure was found to be essentially tetrahedral however the small orthorhombic distortion was also mapped for several polycrystalline samples, with $b / a=0.995$ reported in [7].

Layering of the crystal structure of $\mathrm{RuSr}_{2} \mathrm{GdCu}_{2} \mathrm{O}_{8}$ determines effective separation of the electronically active subsystems of the $\mathrm{Cu}-\mathrm{O}$ and $\mathrm{Ru}-\mathrm{O}$ layers, leading to possibility of tuning their properties with charge doping (note an expectancy for presence of the internal interlayer charge transfer in this structure). Thus, learning of the charge 
carrier concentrations in electronic bands of the $\mathrm{Ru}-\mathrm{O}$ and $\mathrm{Cu}-\mathrm{O}$ layers, and their temperature dependences, seems of primary importance for understanding complex properties of this compound. Next should be considered influence of eventual local structure irregularities, including the interlayer mutual ionic substitution effects, which may also alter the macroscopic properties of investigated samples-this will be commented in a later section of this text. Concerned with the model layered structure of $\mathrm{RuSr}_{2} \mathrm{GdCu}_{2} \mathrm{O}_{8}$, reference [8] presents interesting model, which bases in the consideration of exchange interactions with dominant ferromagnetic $(I)$ and antiferromagnetic (J) character, which are present in the separately treated correlated electron subsystems of the $\mathrm{Ru}-\mathrm{O}$ and $\mathrm{Cu}-\mathrm{O}$ layers, respectively. Structural similarity of the block of $\mathrm{RuO}_{6}$ octahedra to the structure of $\mathrm{Sr}_{2} \mathrm{RuO}_{4}$ compound, even taking into account more isotropic character of the latter, leads to similarities in the component $d$-bands. With similar overlapping planar $\left(d_{x-y}-p\right) \pi$-orbitals in the $\mathrm{Ru}$-O layers, the model assumes then similar parameters of the exchange interaction leaving difference in resulting magnetic and superconducting properties of the $d$ electron bands to difference in the charge doping. Such $t-I$ model written for the Ru-O structural subsystem led to stabilization of either the ferromagnetic or p-type $(l=1)$ superconducting phases depending on comparatively minor modification of charge concentration within the range expected to contain both compounds. Separate consideration for the electron subsystem of the $\mathrm{Cu}-\mathrm{O}$ slabs, for there dominance of the $J_{\gg} I$ condition leads to expectation for superconducting pairing which is of $d_{x 2-y 2}$ singlet type $(l=2)$ in analogy to other cuprates. The assumption of microscopic coexistence of such superconducting and ferromagnetic phases led to proposition of spatial modification of the superconducting order parameter with its phase being shifted in between neighbouring superconducting layers to become nodal within intertwined ferromagnetic slabs of the structure. The interesting experimental results concerned with possible microscopic coexistence of magnetism and superconductivity in the $\mathrm{Ru}-\mathrm{O}$ layers where reported for the sister compound $\mathrm{RuSr}_{2} \mathrm{YCu}_{2} \mathrm{O}_{8}$, for which both spin-lattice relaxation rates determined in the Ru-NMR (at zero field) and Cu-NMR (at $H_{\text {ext }}=100 \mathrm{kOe}$ ) experiments were found to decrease at the compound $T_{c}$, which is characteristic for opening the superconducting gaps, then both in the $\mathrm{Cu}-\mathrm{O}$ and $\mathrm{Ru}-\mathrm{O}$ structural slabs. This result provided argument for presence of the cuprate characteristic superconductivity in the $\mathrm{Cu}-\mathrm{O}$ planes but also for superconductivity coexisting with magnetism in the Ru-O layers, at least at the external magnetic field of that experiment [9]. Analogous experiments performed for the suitable single crystals, when such would become available, seem to be highly awaited.

In the magnetically ordered state below $T_{m}$ the magnetic field dependencies of magnetisation for all $\mathrm{RuSr}_{2} \mathrm{RECu}_{2} \mathrm{O}_{8}$ compounds become hysteretic resembling the behaviour expected for a weak ferromagnet. For $\mathrm{RuSr}_{2} \mathrm{GdCu}_{2} \mathrm{O}_{8}$, the magnetisation below $T_{m}$ appears to predominantly reflect response of the sublattice of large $\mathrm{Gd}^{3+}$ moments $\left(\sim 7 \mu_{B}\right)$ which was also considered to amplify the magnetic characteristics of underlying magnetic order of other magnetic ions [10]. On further lowering the temperature the sublattice of $\mathrm{Gd}^{3+}$ moments orders antiferromagnetically at $2.5 \mathrm{~K}$ [11] much to resemble the $\mathrm{RE}$ magnetic order in $\mathrm{REBa}_{2} \mathrm{Cu}_{3} \mathrm{O}_{7}$ compounds. Our recent measurements of magnetic field dependencies of the isothermal magnetocaloric coefficient for ceramic sample of $\mathrm{RuSr}_{2} \mathrm{GdCu}_{2} \mathrm{O}_{8}$ (value of the coefficient is proportional to field 
driven changes of the magnetic entropy) signals the response of a complex magnetic system, and appears to involve the magnetic correlations present in spatially constrained range [12]. Such response may be referred to consequence of magnetic phase separation, which was proposed to remain inherent characteristic of the $\mathrm{Ru}-1212$ ruthenocuprates [13]. Soon after the discovery of the combined magnetic and superconducting characteristics for $\mathrm{RuSr}_{2} \mathrm{GdCu}_{2} \mathrm{O}_{8}$, the zero field $\mu \mathrm{SR}$ (muon spin rotation) measurements provided with the temperature dependence of the muon sensed internal field associated with the Ru ordered state, which shows the magnetisation dependence expected value of $T_{m}$ and no modification of the internal field at low temperatures upon entering the temperature range of diamagnetic shielding in the sample [14]. The observed increase of the transverse relaxation rate (for part of the signal which originates in decay of muons with precessing spins) below $T_{c}$ provides for increased distribution of the muon spin sensed internal field, whereas the unusually fast longitudinal relaxation, which was registered in broad temperature range below $T_{m}$ seem to indicate that also other magnetic sublattices, likely of the $\mathrm{Gd}^{3+}$ moments, become involved in dynamics of the muon spin lattice relaxation. The experiment also established minimal volume fraction to be associated with the observed internal field at approx. $80 \%$ of the sample volume, thus providing for its bulk origin.

The magnetic ground state of the $\mathrm{Ru}$ sublattice in $\mathrm{RuSr}_{2} \mathrm{GdCu}_{2} \mathrm{O}_{8}$ was approached with several different models which originated in interpretation of several different experiments. Due to lack of suitable single crystals most of these were based in the investigation of sintered samples, which added complexity to the interpretation. Early neutron powder diffraction (NPD) served to conclude the G-type antiferromagnetic order of the Ru moments with easy axis pointing along the $c$ direction, i.e. perpendicular to the $\mathrm{CuO}_{2}$ planes, and ad hoc associated an eventual presence of the ferromagnetic component to possibility of the field dependent canting of the $\mathrm{Ru}^{5+}$ magnetic moments $[4,11]$. The analysis of the field dependent magnetic diffraction (also performed for samples prepared with use of $\mathrm{Gd}^{160}$ isotope for lowering the neutrons scattering cross-section) for assuming such model pointed to consequence of the considerable field induced increase of the canting angle, estimated at more than $75^{\circ}$ for $H_{\text {ext }}>15 \mathrm{kOe}$ [5]. The NPD then provided evidence for presence of substantial ferromagnetic component within the $a-b$ planes of the structure at the magnetic field considerably lower from the second critical field of the superconducting phase. Also measurements of the Ru-NMR (nuclear magnetic resonance) for zero-field and the field dependent spectra provided arguments for involving in description substantial inplane magnetisation of the Ru moments, however present at much lower field values. Established magnetic field dependences of the resonance frequencies led to proposition of the type-I AFM structure built of ferromagnetic $a-b$ planes, with spin flop transition or field induced rotation of the in-plane magnetisation vectors, which are both probable for expectation of substantial anisotropy of the magnetic system [15]. This and several other experiments evidenced presence of two valence states, 5+ and 4+, for the $\mathrm{Ru}$ ions [15-18] with expectation of magnetic itinerancy for the $\mathrm{Ru}^{4+}$ subset which based in the form of measured NMR signal [15]. Investigation of the Ru magnetic ground state in $\mathrm{RuSr}_{2} \mathrm{GdCu}_{2} \mathrm{O}_{8}$ performed for the single crystal was so far communicated only by means of the resonant X-ray diffraction with photon energy matching the $L_{2}$ absorption edge of $\mathrm{Ru}$ [19]. The results provide with some reconciliation for the 
previously formulated models proposing the G-type magnetic structure albeit with the $\mathrm{Ru}$ moments pointing along the low symmetry (102) direction, i.e. allowing for presence of considerable out-of plane and in-plane components in the Ru-O layers, and the in-plane component stacked antiparallely along the $c$ direction. Other than only the $\mathrm{Ru}$ subsystem magnetic ordering was proposed for the Ru-1212 structure based on the neutron diffraction investigation of the $\mathrm{Y}$ based compound. This compound, although not straightforward to synthesise (the 1212-type phases of ruthenocuprates with rare earth ions smaller than Gd require the high pressure conditions of high temperature solid state reaction) appears more suitable for the NPD measurement due to small neutron scattering by $\mathrm{Y}$ ions. These measurements for the superconducting sample with nominal composition $\mathrm{Ru}_{0.9} \mathrm{Sr}_{2} \mathrm{YCu}_{2.1} \mathrm{O}_{8}$ concluded G-type antiferromagnetic ordering in the Ru sublattice with comparable $T_{m}=135 \mathrm{~K}$, however, the observed low temperature build up of intensity for one of the low angle nuclear peaks was assigned to presence of magnetic ordering in the $\mathrm{Cu}-\mathrm{O}$ planes [20]. This interpretation followed in Ref. [21] providing with hypothesis that an apparent ferromagnetic component, which is present in the compound magnetisation appears rather in result of the interaction between magnetically ordered $\mathrm{Cu}$ and $\mathrm{Ru}$ sublattices. The superconducting diamagnetic response observed at low temperatures was associated to the consequence of hole doping enhanced for likely occurrence of a slight $\mathrm{Cu} \rightarrow \mathrm{Ru}$ heterovalent substitution. In view of the proposed complex component magnetism furthering of such interpretation, which was supported also in communicated magnetic characteristics of $\mathrm{Ru}_{0.9} \mathrm{Sr}_{2} \mathrm{YCu}_{2.1} \mathrm{O}_{8}$, would clearly benefit from experimentally accessing the temperature dependence of anisotropy of the compound's magnetisation. Potential complexity may also arise from the sample homogeneity issues, as indicated by the authors noted broadening of there analysed nuclear reflection.

Investigations of the $\mathrm{Ru}$ magnetic ordering in ruthenocuprates often concern also other type of compounds described with the formula $\mathrm{RuSr}_{2} \mathrm{RE}_{2-\mathrm{x}} \mathrm{Ce}_{\mathrm{x}} \mathrm{Cu}_{2} \mathrm{O}_{10-\mathrm{y}}$ (mostly investigated were phases with $\mathrm{RE}=\mathrm{Eu}, \mathrm{Gd}, \mathrm{Y}$ ). The crystal structure of this so called Ru1222-type is similar to that of $\mathrm{RuSr}_{2} \mathrm{RECu}_{2} \mathrm{O}_{8}$ with the single $\mathrm{RE}$ layer replaced by the $\left(\mathrm{RE}_{2-\mathrm{x}} \mathrm{Ce}_{\mathrm{x}}\right) \mathrm{O}_{2}$ fluorite-type layer with consequence of shifting the structural blocks above and below by vector $(1 / 2,1 / 2,0)$ (more detailed description will yet involve complex structural tilts, for references see i.a. in [22]). The fluorite-type layer supports variable oxygen occupancy, denoted with the parameter $y$ in the above formula. Elongation of the unit cell in respect to the Ru1212-type results with longer distance between the neighbouring layers of $\mathrm{RuO}_{6}$ octahedra providing with expectation of larger $c$ vs. $a-b$ magnetic anisotropy for the system of Ru moments. The temperature dependencies of magnetisation indicated that the magnetic properties are here more complex than for $\mathrm{RuSr}_{2} \mathrm{RECu}_{2} \mathrm{O}_{8}$. There are two characteristic temperatures introduced for description of the magnetically ordered state, $T_{m}>T_{i r r}$ (for solid solution $\mathrm{Eu}_{2-\mathrm{x}} \mathrm{Ce}_{\mathrm{x}} \mathrm{RuSr}_{2} \mathrm{Cu}_{2} \mathrm{O}_{10-\mathrm{y}} T_{i r r}=80 \mathrm{~K}-120 \mathrm{~K}$ and $T_{m}=125 \mathrm{~K}-165 \mathrm{~K}$, where the ranges reflect their dependence on the $x$ and $y$ parameters [23,24]). At temperatures below $T_{i r r}$, the compounds magnetisation becomes strongly field hysteretic, which was interpreted for weak ferromagnetism, with the substantial FC vs. ZFC (field-cooled vs. zerofield cooled) irreversibility possibly coming in result of the domain structure. The superconducting phase was there observed with suitable $\mathrm{Ce}$ and oxygen concentrations [23,25] with a maximum $T_{c}^{o n} \approx 52 \mathrm{~K}$ reported for $\mathrm{RuSr}_{2} \mathrm{Gd}_{1.5} \mathrm{Ce}_{0.5} \mathrm{Cu}_{2} \mathrm{O}_{10-y}$, i.a. in [26]. 
To the best of my knowledge, single crystals of the Ru1222-type were not reported. Then for expected anisotropy of the magnetic properties one needs to reference to properties of the high quality, $c$ axis oriented epitaxial thin films of $\mathrm{RuEu}_{1.5} \mathrm{Ce}_{0.5} \mathrm{Sr}_{2-}$ $\mathrm{Cu}_{2} \mathrm{O}_{10-\mathrm{d}}$, which were grown by the flux assisted solid phase epitaxy technique [27]. For these films, the hard axis of magnetisation was found to place along the $c$ axis, and no magnetic anisotropy was detected within the $a b$ plane. Since properties of thin films may easily differ from those of a bulk single crystal (for example for the substrate caused strain) it should be noted that reported temperature dependences of both components of magnetisation were found to be qualitatively similar to the data collected for bulk samples [27]. The anomalous features of dynamic magnetic characteristics found in the limit of low magnetic field for bulk samples of $\mathrm{RuEu}_{2-\mathrm{x}} \mathrm{Ce}_{\mathrm{x}} \mathrm{Sr}_{2} \mathrm{Cu}_{2} \mathrm{O}_{10-\mathrm{d}}$ [28] led to conclude a meta-stable character of the accessed magnetic state. The ground state model proposed in [28] preserved the G-type AFM ordering in consistency with prior works, and based on the postulated canting of the Ru spins, however, here in an essentially random direction off the $c$ axis. The magnetic characteristics were explained with presence of a weak magnetic field-driven flop transition of the inplane spin components. Larger distance between the magnetic layers than in the Ru1212-type structure led to consider for a role of weak dipolar interaction between adjacent $\mathrm{Ru}$-O layers. Then, the antiparallel alignment of nearest in-plane components of the magnetic moments in neighbouring layers could become overcome with very weak external field $[27,28]$. Such a model seems to explain also other unusual magnetic behaviour reported for the Ru-1222 compounds as still controversial presence of FM/ AFM phase separation [29] or the spin glass type magnetic characteristics. Reported in many research papers the complex macroscopic characteristics of magnetic and superconducting states called for further microscopic investigation of spatial homogeneity and phase separation in the samples. Here come the results of muon spin rotation spectroscopy $(\mu \mathrm{SR})$ collected for the polycrystalline $\mathrm{RuEu}_{1.4} \mathrm{Ce}_{0.6} \mathrm{Sr}_{2} \mathrm{Cu}_{2} \mathrm{O}_{10}$, which provided with analysis of the volume fractions associative to internal magnetic field observed in a broad range of temperatures [30]. It was shown that in the temperature range $T_{i r r}<\mathrm{T}<T_{m}$ the magnetic phase accounts only for approximately $15 \%$ of the sample volume, however below $\sim 78 \mathrm{~K}$, which also marks a sudden, diamagnetic like, drop of the ZFC branch of magnetisation, the sample becomes magnetic in its whole volume. For there estimated limit of secondary phases, such results suggested presence of magnetic clusters within the Ru1222 phase, at least for higher temperature data. With this conclusion it should be noted that the characteristics of some from the comparable Ru1222-type samples were found to differ and thus more universal picture should be built cautiously. The analysis of neutron powder diffraction and polarized neutron transmission data collected for the superconducting $\left(T_{c}=35 \mathrm{~K}\right) \mathrm{RuSr}_{2} \mathrm{Eu}_{1.2-}$ $\mathrm{Ce}_{0.8} \mathrm{Cu}_{2} \mathrm{O}_{10}$ concluded that the observed magnetic signals do not originate in the Ru1222-type phase, although complexity of that sample did not allow to relate the signal with any particular minority phase [31]. On the other hand, recently communicated results of the XMCD spectroscopy (X-ray Magnetic Circular Dichroism) at $5 \mathrm{~K}$ for the superconducting sample of $\mathrm{RuSr}_{2} \mathrm{Eu}_{1.5} \mathrm{Ce}_{0.5} \mathrm{Cu}_{2} \mathrm{O}_{10}$ concluded the presence of ferromagnetic moment at $0.21 \mu_{B} / \mathrm{Ru}$, whereas the accompanying results of EXAFS spectroscopy (Extended X-ray Absorption Fine Structure) excluded presence of secondary phases, at least such for which the coordination of Ru ions is markedly distinct from 
that in the main phase [32]. Although the investigated samples superconduct at low temperatures, extending on the argument of microscopic coexistence of this superconductivity with the material's magnetism should come only after estimation of both associated volumes. The alterations of the local structure reported for the Ru1222 phase include the embedded inclusions of Ru1212 phase [33].

Not only magnetic, but also the superconducting characteristics of ruthenocuprates led for considering the material inherent phase separation, with noted presence of weak links in the granular structure of investigated samples which may effectively enhance the signatures of underlying phase inhomogeneity-for extensive discussion see [13] where for $\mathrm{RuSr}_{2} \mathrm{RECu}_{2} \mathrm{O}_{8}$ was proposed the model of separation to an ensemble of the antiferromagnetic/superconducting and ferromagnetic/non-superconducting domains. The magnetic penetration depth for these samples with the characteristic grain size below $5 \mu \mathrm{m}$ was estimated at $\lambda(0 K)=4 \mu \mathrm{m}$ [13]. Such large estimated value becomes, however, justified for postulated presence of the nano-size ferromagnetic domains, which separate the superconducting regions in the crystal structure [13]. Then, measured $T_{c}$ would assume a role of effective temperature of the phase coherence on the array of Josephson junctions, with $\lambda$ becoming a measure of its temperature dependence. As natural would then come the observed large differences in $T_{c}$ for only slightly different, and nominally same stoichiometric, samples. For $\mathrm{RuSr}_{2} \mathrm{Gd}$ $\mathrm{Cu}_{2} \mathrm{O}_{8}$, the maximum of the transition onset was observed at $52-55 \mathrm{~K}$, with that high values being characteristic also for all of the first reported small single crystals (200 $\mu \mathrm{m} * 200 \mu \mathrm{m} * 50 \mu \mathrm{m}$ ) [34]. Among so far few characteristics communicated for these crystals are $I-V$ dependencies interpreted for presence of the Josephson type coupling along the $c$ axis, so and for the effectively layered structure built of interlaced SC-I-SC slabs [35]. In this transport experiment no specific features were associated to presence of the Ru sublattice magnetism (note that qualitatively similar Josephson characteristics have been observed for several other underdoped HTSC cuprates of sufficiently large structural anisotropy i.e. Bi-2212, Tl-2223, see [36] and therein). For the same batch single crystals recently reported results of the resonant X-ray diffraction [19] (commented earlier for investigation of the magnetic ground state of $\mathrm{RuSr}_{2} \mathrm{GdCu}_{2} \mathrm{O}_{8}$ ) concluded likely presence of substantial in plane component magnetisation in the $\mathrm{RuO}_{2}$ layers. Other interesting feature of the reported crystals is that the temperature of magnetic transition was found substantially lowered in respect to that observed in the polycrystalline samples- $T_{m} \approx 102 \mathrm{~K}$ was inferred from the temperature dependence of the intensities of two resonant RXD magnetic reflections, and supported in data of the angle dependent temperature dependencies of magnetisation [19]. Simultaneously observed upturn of the magnetic susceptibility at approximately $40 \mathrm{~K}$ above such determined $T_{m}$ was there plausibly assigned to presence of non-compensated ferromagnetic moments, which might arise locally due to structural modifications in the crystal [19]. It seems, however, possible that the transition at $T_{m} \approx 102 \mathrm{~K}$ comes preceded by lower dimensional magnetic correlations set at substantially higher temperature and arising uniformly in the crystal volume. Overall, the reviewed results may signal that even larger extent structural alternations seems to be present in so far accessed single crystals than in the polycrystalline material. Such idea also comes in context of the investigation of local structure in the polycrystalline superconducting sample of $\mathrm{RuSr}_{2} \mathrm{GdCu}_{2} \mathrm{O}_{8}$ [37]. The high-resolution transmission electron microscopy 
(HRTEM) and the high-resolution scanning transmission microscopy data revealed presence of locally inhomogeneous structure built of the nanometre range intergrowths of the 1212-type and of the second phase with doubled $c$ axis parameter for replacing every second $\mathrm{Ru}-\mathrm{O}$ layer with the $\mathrm{Cu}-\mathrm{O}$ chain layer-in resemblance of the layering alignment in $\mathrm{REBa}_{2} \mathrm{Cu}_{3} \mathrm{O}_{7}$ structure. It was then postulated it could be of such structural inclusions to support the superconducting phase in the material [37]. What makes such conclusion less intuitive are postulated presence of the bulk Meissner effect in analogous ceramic samples of $\mathrm{RuSr}_{2} \mathrm{GdCu}_{2} \mathrm{O}_{8}$ [38] and in the high pressure synthesised samples of $\mathrm{RuSr}_{2} \mathrm{YCu}_{2} \mathrm{O}_{8}$ [39]. On the other hand, within the phase separation scenario proposed in [13] the Meissner state would not be expected. Other local structural features mapped in the HRTEM investigation [37] include the $90^{\circ}$ rotations and the antiphase boundaries. Note, that for its close proximity to the $a=b=c / 3$ condition (for elementary cell parameters), the $\mathrm{RuSr}_{2} \mathrm{GdCu}_{2} \mathrm{O}_{8}$ may indeed become vulnerable to form the intergrowths of $90^{\circ}$ rotated structural cubes, in similar to the micro-domain texture reported for the 1212-type iridocuprates [40]. Such structural domains may also be envisioned to play a role in supporting the phase separated superconductivity, as in principle could do a network of the associated magnetic domain boundaries-such possibilities, however, at present remain only speculative.

In discussion of possible ways for accommodating the superconducting coherence in $\mathrm{RuSr}_{2} \mathrm{GdCu}_{2} \mathrm{O}_{8}$ one needs to turn to implications of the finding that same nominally stoichiometric compound was also found non-superconducting [3,41,42], and that for quite high quality samples, which do not unequivocally differ in their diffraction investigated crystal structure or in estimated oxygen content [5]. It seemed therefore important to further investigate for the features, which control the superconducting $T_{c}$, then only re-accessing twining of the compound superconductivity with its magnetism. Turning to solid state chemistry of the sintered samples of ruthenocuprates note here recently published investigation for the Ru sublimation processes, likely accompanying the high temperature reaction at oxidative conditions, which indicated differences in the $\mathrm{Ru}$ content in the bulk vs. surface of sintered $\mathrm{RuSr}_{2} \mathrm{GdCu}_{2} \mathrm{O}_{8}$ and associated the effect to observed changes of $T_{c}$ [43] (for extensive review of related synthesis issues reported by the same group see Ref. [44]). It should be, however, noted that currently there is no compelling evidence of any particular form of the sublimation associated, so and probably non-uniform, structural alternations of the $\mathrm{Ru}-1212$ phase. Also note, that the superconducting state may be influenced not only by associated charge doping or the impurity caused deterioration of the condensate coherence, but also with modification of the compound magnetism. Then, potential significance of local modifications in the $\mathrm{Ru} / \mathrm{Cu}$ ratio in $\mathrm{RuSr}_{2} \mathrm{GdCu}_{2} \mathrm{O}_{8}$ samples comes in result of observations of increased $T_{c}$ in the $\mathrm{Ru}_{0.9} \mathrm{Sr}_{2} \mathrm{GdCu}_{2.1} \mathrm{O}_{8}$ vs. nominally stoichiometric sample [45] and even larger increase of $T_{c}$ observed upon further $\mathrm{Cu} \rightarrow \mathrm{Ru}$ substitution achieved in the compounds synthesised at high pressure of oxygen [46]. Possibility of the $\mathrm{Ru} / \mathrm{Cu}$ defect ordering was early suggested based on the electron diffraction observed superstructure in the superconducting sample of $\mathrm{RuSr}_{2} \mathrm{GdCu}_{2} \mathrm{O}_{8}$ [47]. For the local structure described with formula $\mathrm{Ru}_{1-\mathrm{x}} \mathrm{Sr}_{2} \mathrm{GdCu}_{2+\mathrm{x}} \mathrm{O}_{8-\mathrm{d}}, \times>0$, the $T_{c}$ would be expected to depend on differences in structure's oxygen content [42] in resembling the oxygen content driven charge doping in structurally related $\mathrm{REBa}_{2} \mathrm{Cu}_{3} \mathrm{O}_{7-\mathrm{y}}$. If such structural modifications would occur only locally or at the domain boundaries with constrained 
dimensions, the conventional methods of detecting modification of the oxygen content may well become insufficient. Our recent experimental approach based in comparing the superconducting and magnetic properties for the samples which were prepared at the same conditions but with slightly modified nominal stoichiometry [22]- $\mathrm{RuSr}_{2} \mathrm{Gd}$ $\mathrm{Cu}_{2} \mathrm{O}_{8}$ and $\mathrm{Ru}_{0.98} \mathrm{Sr}_{2} \mathrm{GdCu}_{2} \mathrm{O}_{8}$, aiming at then induced differences in their physical properties. The structural alterations of the $\mathrm{Ru}-1212$ phase, if there achieved, would not necessarily match its nominal stoichiometry. Since the syntheses were conducted at high temperature close to the melting point for this phase, most affected may indeed become local regions characterised by increased Ru sublimation. Further remarks on involved synthesis process were presented in [12]. Observed slight and reproducible shift of the magnetic ordering temperature $\left(T_{m} \approx 126 \mathrm{~K} v s .132 \mathrm{~K}\right.$ for $\times=0.02 v s . \times=$ $0, \mathrm{Ru}_{1-\mathrm{x}} \mathrm{Sr}_{2} \mathrm{GdCu}_{2} \mathrm{O}_{8}$ ) may well result in dilution of the $\mathrm{Ru}$ magnetic sublattice [48]. The resistivity and magnetically determined onset of the superconducting transition was found in such samples considerably higher for the increased nominal Ru deficiency $\left(T_{c}^{o n} \approx 50 \mathrm{~K} v s .40 \mathrm{~K}\right.$ for $\times=0.02$ vs. $\left.\times=0\right)$. Comparison of the $a c$ susceptibility measured diamagnetic response at low temperatures revealed also that for the $\times=0.02$ sample $T_{c}{ }^{o n}$ becomes much less dependent on the ripping field amplitude for its limiting small values, which suggest sensing response of the superconducting regions with more constrained dimensionality for the $x=0$ sample. Since the temperature onset of resistive transition coincides there with insignificant increase in the susceptibility signal [22], it suggests that the self fields that come in result of the screening currents (first intra-crystalline paths are formed at that temperature) are involved in inducing an unscreened response which is observed as an extra magnetic component. This effect may be taken further for discussing the most probably constrained dimensionality of the superconducting phase in such samples [48]. Differences in the magnetic field dependence of the temperatures characteristic for resistive superconducting transitions [48] were also found supportive of more inhomogeneous nucleation of the superconducting phase in nominally stoichiometric $\mathrm{RuSr}_{2} \mathrm{GdCu}_{2} \mathrm{O}_{8}$ sample.

In conclusion, aside of recently investigated material issues, which may involve not enough controlled local non-stoichiometry effects, what appears promising in the evolving field is that ruthenocuprates seem carrying the potential for investigation of the cuprates characteristic superconductivity in its incipient, effectively quasi two dimensional, limit. This comes for the crystal structure quite similar to well known $\mathrm{REBa}_{2} \mathrm{Cu}_{3} \mathrm{O}_{7-\mathrm{x}}$ superconductor, but which seems to provide for the effective decoupling of structural layers supporting the quasi two dimensional superconducting condensate. The fundamental properties of these compounds could, however, become far more accessible with suitably property tuned, high quality research samples. On high demand appear especially single crystals, which are not superconducting, and such which will support the superconducting phase induced in a charge carrier doping controlled manner. Such materials should be of benefit also for continuing studies of the magnetic ground state. The field then could be carried forward most effectively by furthering the synthesis effort.

Received: 19 March 2010 Accepted: 13 July 2010 Published: 13 July 2010

References

Bauernfeind L, Widder W, Braun HF: Physica C 1995, 254:151.

2. Bauernfeind L, Widder W, Braun HF: J Low Temp Phys 1996, 105:1605. 
3. Felner I, Asaf U, Reich S, Tsabba Y: Physica C 1999, 311:163.

4. Chmaissem O, Jorgensen JD, Shaked H, Dollar P, Tallon JL: Phys Rev B 2000, 61:6401.

5. Blake GR, Radaelli PG, Jorgensen JD, Klamut PW, Dabrowski B, Chmaissem O: Poster Presented at the European Conference on Neutron Scattering Montpellier, France 2003.

6. McLaughlin AC, Zhou W, Attfield JP, Fitch AN, Tallon JL: Phys Rev B 1999, 60:7512.

7. Martinelli A, Artini C, Cimberle MR, Costa GA, Ferretti M, Masini R, Mele P: Phys Rev B 2004, 69:052507.

8. Kuz'min EV, Ovchinnikov SG, Baklanov IO, Goryachev EG: J Exp Theor Physics 2000, 91:353.

9. Tokunaga Y, Kotegawa H, Ishida K, Kitaoka Y, Takagiwa H, Akimitsu J: Phys Rev Lett 2001, 86:5767.

10. Jorgensen JD, Chmaissem O, Shaked H, Short S, Klamut PW, Dabrowski B, Tallon JL: Phys Rev B 2001, 63:54440.

11. Lynn JW, Keimer B, Ulrich C, Bernhard CH, Tallon JL: Phys Rev B 2000, 61:R14964.

12. Klamut PW, Plackowski T: Supercond Sci Technol 2009, 22:025021.

13. Lorenz B, Xue YY, Chu CW: Studies of High-Temperature Superconductors (New York: Nova Science)Narlikar AV 2004, 46

14. Bernhard C, Tallon JL, Niedermayer CH, Blasius TH, Golnik A, Bruecher E, Kremer RK, Noakes DR, Stronach CE, Ansaldo E: J Phys Rev B 1999, 59:14099.

15. Han ZH, Budnick Jl, Hines WA, Klamut PW, Maxwell M, Dabrowski B: J Magn Magn Mater 2006, 299:338.

16. Liu RS, Jang L-Y, Hung H-H, Tallon JL: Phys Rev B 2001, 63:212507.

17. Papageorgiou TP, Casini E, Skourski Y, Herrmannsdörfer T, Freudenberger J, Braun HF, Wosnitza J: Phys Rev B 2007, 75:104513.

18. Kumagai K, Takada S, Furukawa Y: Phys Rev B 2001, 63:180509.

19. Bohnenbuck B, Zegkinoglou I, Strempfer J, Nelson CS, Wu H-H, Schüßler-Langeheine C, Reehuis M, Schierle E, Leininger PH, Herrmannsdörfer T, Lang JC, Srajer G, Lin CT, Keimer B: Phys Rev Lett 2009, 102:037205.

20. Yelon WB, Cai Q, Lamsal J, Blackstead HA, Kornecki M, Awana V, Kishan H, Balamurugan S, Takayama-Muromachi E: J Appl Phys 2007, 101:09G104.

21. Blackstead HA, Yelon WB, Kornecki M, Smylie MP, Cai Q, Lamsal J, Awana VPS, Balamurugan S, Takayama-Muromachi E: Phys Rev B 2007, 76:094507.

22. Klamut PW: Supercond Sci Technol 2008, 21:093001.

23. Felner I, Asaf U, Galstyan E: Phys Rev B 2002, 66:024503.

24. Felner I, Galstyan E, Nowik I: Phys Rev B 2005, 71:064510.

25. Mclaughlin AC, Attfield JP, Asaf U, Felner I: Phys Rev B 2003, 68:014503.

26. Felner I, Asaf U, Ritter F, Klamut PW, Dabrowski B: Physica C 2001, 364-365:368.

27. Lu WQ, Yamamoto Y, Petrykin W, Kakihana M, Matsumoto Y, Joshi US, Koinuma H, Hasegawa T: Thin Solid Films 2005, 486:79.

28. Živković I, Hirai Y, Frazer BH, Prester M, Drobac D, Ariosa D, Berger H, Pavuna D, Margaritondo G, Felner I, Onellion M: Phys Rev B 2002, 65:144420.

29. Felner I, Awana VPS, Takayama-Muromachi E: Phys Rev B 2003, 68:094508.

30. Shengelaya A, Khasanov R, Eshchenko DG, Felner I, Asaf U, Savić IM, Keller H, Müller KA: Phys Rev B 2004, 69:024517.

31. Lynn JW, Chen Y, Huang Q, Goh SK, Williams GVM: Phys Rev B 2007, 76:014519.

32. Souza-Neto NM, Haskel D, Lang JC, Chmaissem O, Dabrowski B, Felner I: Phys Rev B 2009, 80:140414(R).

33. Asthana A, Matsui Y: Physica C 2008, 468:458.

34. Lin CT, Liang B, Ulrich C, Bernhard C: Physica C 2001, 364-365:373.

35. Nachtrab T, Koelle D, Kleiner R, Bernhard C, Lin CT: Phys Rev Lett 2004, 92:117001.

36. Yurgens A: Supercond Sci Technol 2000, 13:R85.

37. Lebedev OI, Van Tendeloo G, Attfield JP, Mclaughlin AC: Phys Rev B 2006, 73:224524.

38. Bernhard CH, Tallon JL, Brücher E, Kramer RK: Phys Rev B 2000, 61:R14960.

39. Awana VPS, Kawashima T, Takayama-Muromachi E: Phys Rev B 2003, 67:172502.

40. Dos Santos-Garcia AJ, Aguirre MH, Morán E, Puche RS, Alario-Franco MÁ: J Solid State Chem 2006, 179:1296.

41. Zhigadlo ND, Odier P, Marty J CH, Bordet P, Sulpice A: Physica C 2003, 387:347.

42. Klamut PW, Dabrowski B, Mini SM, Maxwell M, Mais J, Felner I, Asaf U, Ritter F, Shengelaya A, Khasanov R, Savic IM, Keller H, Wiśniewski A, Puźniak R, Fita IM, Sulkowski C, Matusiak M: Physica C 2003, 387:33.

43. Casini E, Kempf M, Krämer J, Braun HF: J Phys Condens Matter 2009, 21:254210.

44. Braun H, Bauernfeind L, Korf O, Papageorgiou TP: Ruthenate and Rutheno-Cuprate Materials Unconventional Superconductivity, Magnetism and Quantum Phase Transitions (Springer Lecture Notes in Physics) (Berlin: Springer)Noce C, Vecchione A, Cuoco M and Romano A 2002, 603.

45. Sader E, Matveev AT, Habermeier H-U: Supercond Sci Technol 2006, 19:L29,

46. Klamut PW, Dabrowski B, Koleśnik S, Maxwell M, Mais J: Phys Rev B 2001, 63:224512.

47. Awana VPS, Ichihara S, Nakamura J, Jarppinen M, Yamauchi H, Yang J, Yelon WB, James WJ, Malik SK: J Appl Phys 2002, 91:8501.

48. Klamut PW, Plackowski T, Matusiak M: J Low Temp Phys 2010, 159:576.

doi:10.1186/1754-0429-3-2

Cite this article as: Klamut: Commentary on the superconducting and magnetic properties of the

ruthenocuprates. PMC Physics B 2010 3:2. 